\title{
Perceptions of therapeutic principles in a therapeutic community
}

\begin{tabular}{|r|l|}
\hline Journal: & $\begin{array}{l}\text { Therapeutic Communities: The International Journal of Therapeutic } \\
\text { Communities }\end{array}$ \\
\hline Manuscript ID & TC-02-2017-0009.R1 \\
\hline Manuscript Type: & Academic Paper \\
\hline Keywords: & $\begin{array}{l}\text { personality disorder, learning disability, forensic, secure, Therapeutic } \\
\text { communities, intellectual disability }\end{array}$ \\
\hline \multicolumn{2}{|l}{} \\
\hline
\end{tabular}

\section{SCHOLARONE ${ }^{\text {M }}$ \\ Manuscripts}


Table 1: Participants demographics - means and ranges

\begin{tabular}{|c|c|c|}
\hline & Service users & Staff \\
\hline Age & $\begin{array}{l}38 \text { years (range 27-50 } \\
\text { years) }\end{array}$ & 42 years (34-53 years) \\
\hline Time spent on LDTC & 4.5 years $(2.5-5$ years $)$ & $\begin{array}{l}5 \text { years (4-5 years and } \\
11 \text { months) }\end{array}$ \\
\hline $\begin{array}{l}\text { Time spent in high } \\
\text { secure hospital }\end{array}$ & 8 years (2.5-13 years) & $N / A$ \\
\hline
\end{tabular}




\section{Table 2: Frequencies of categories endorsed by participants based on} Haigh's quintessence principles

\begin{tabular}{lll}
\hline TC Principles & $\begin{array}{l}\text { Patient responses } \\
\text { (frequency } \\
\text { mentioned and by } \\
\text { how many) }\end{array}$ & $\begin{array}{l}\text { Staff responses } \\
\text { (frequency } \\
\text { mentioned and by } \\
\text { how many staff) }\end{array}$ \\
\hline Attachment & $17(5 / 6)$ & $29(6 / 6)$ \\
\hline Containment & $51(6 / 6)$ & $58(6 / 6)$ \\
\hline Communication & $53(6 / 6)$ & $67(6 / 6)$ \\
\hline Involvement and Inclusion & $35(6 / 6)$ & $63(6 / 6)$ \\
\hline Agency & $46(6 / 6)$ & $78(6 / 6)$ \\
\hline
\end{tabular}




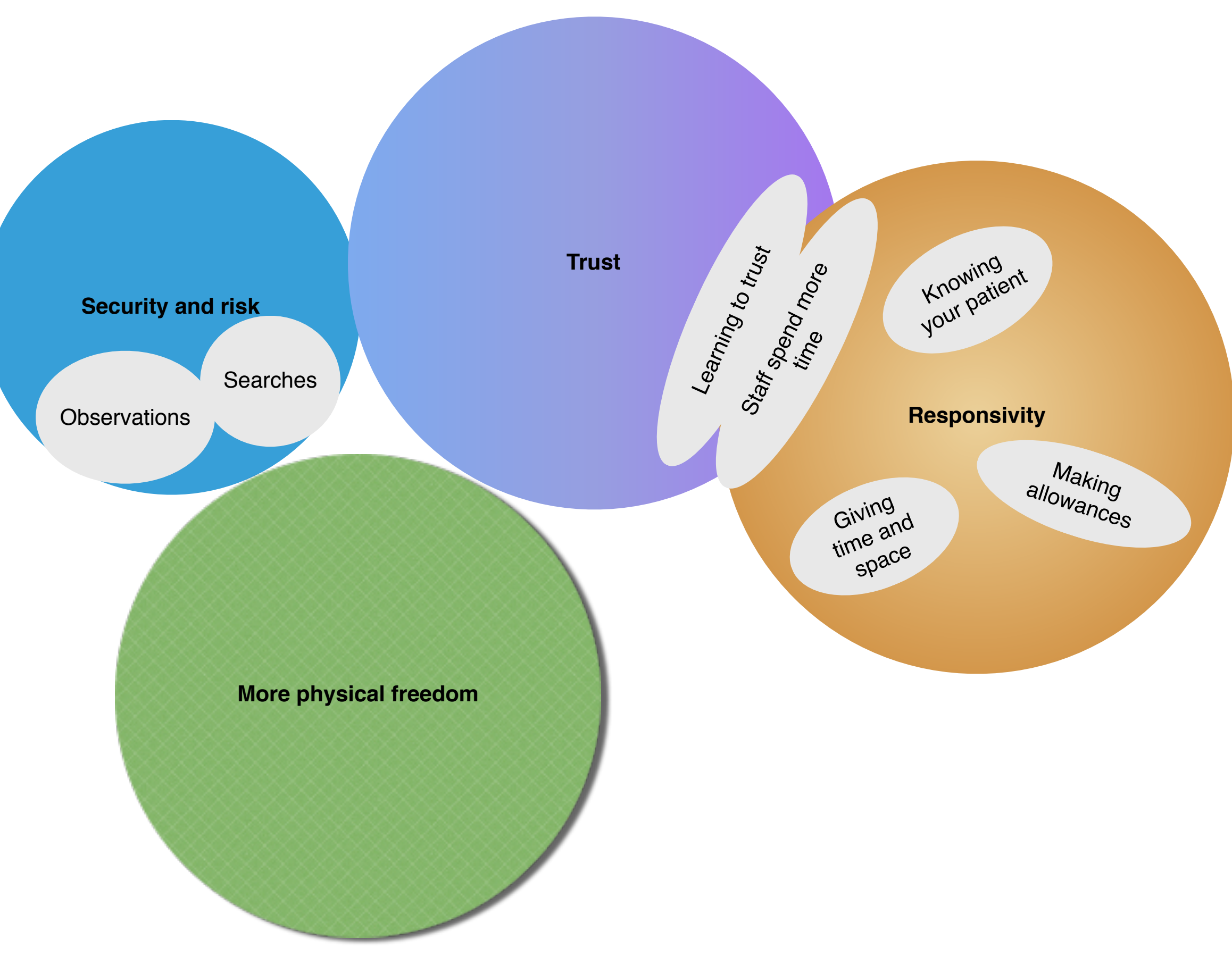




\section{Appendix A Deductive analysis: Example quotes}

\section{Attachment}

Service users:

[...] you feel safe, you feel secure, it just feels like a family.

[...] staff on here treat you like a human being.

Staff:

[...] I mean like I say relationship wise there is a lot stronger bond now between the patients themselves and the staff and patients.

\section{Containment}

Service users:

[...] basically people can help and support you when you go through bad things or hard things.

Staff:

[...] I like that we're not intervening and controlling situations maybe like other wards do to a far greater degree, you tend to feel quite proud that you're not intervening, it's quite difficult because it's not an easy decision not to control, the easy thing to do is to control a situation, manage it, stepping back and having the confidence to step back and take that calculated risk but it is a calculated risk but backed up by a relationship and knowledge of patients so I like that.

\section{Communication}

Service users:

[...] Here the patients ask you questions like why did you do it or can you explain to me what made you do it and you explain it and that.

[...] On here you can tell them what you've done. Yeah, you can talk about your offences on this ward yeah but if you talk about your offences on the [separate ward] then you get beat up. I think it's a good thing because you've got to get it out somehow haven't you...। talk about it often since l've been on here and it's more better for me to talk about it. 
Staff:

[...] I like the fact that they're able to challenge each other...so basically if a patient was to do something wrong or what's perceived as wrong by other people...they get challenged at the time by the staff but then we'll put them in the book, community book, agenda book, so then in a community meeting that obviously gets raised so they get challenged by their peers as well, so it's, obviously the staff will challenge them at the time but it will come about again where they get challenged as well.

[...] but I do think that they believe that they can come to us with anything and talk to us and some of them do open up about some things that they probably wouldn't do anywhere else, so they obviously feel safe enough to do it which is a good thing.

[...] Suppose with the patients, I don't know, just feel more comfortable, more at ease and I think I see it as a two-way thing, you know, with the patients, I think l've got a better relationship with them than previously, just through talking to them more, through working together in the groups, having one-to-one sessions etc., just feel that you've got a stronger relationship with the patients on here than I would have had previously, more opportunity to talk, it's more open, whereas previously it wasn't so encouraged to have that openness twenty-four hours a day, you know, it was more of a do all the therapy off the ward elsewhere with other people and come back and we would never become involved with that side sometimes. 


\section{Involvement and inclusion}

Service users:

[...] When you break a rule you could be day-room bound, you'll go into the community book to talk about it, that would be on a Monday or a Friday group, then it could be taken to your Wednesday group where you could talk about it more in-depth or it depends on the situation.

Staff members:

[...] Depending which rule, if they just don't go to a TC activity they have their room locked off for the rest of the day and they can't attend social functions that day. They're only observed in the day-room if it's kind of medication that they're refusing just obviously as I said about their mental state or if they're bullying, if they're bullying one another the verbal abuse then we'll be with them to monitor that.

\section{Agency}

Service users:

[...] We make the rules on here. It's a good thing.

[...] Mainly staff and us. If we're not happy with something we can put it in the book [community agenda book].

Staff:

[...] It does, yeah it does, they're the first to grass on everybody, it's weird really because they will try it on themselves, you know try and break a rule, but they're the first to come and tell you when somebody else has tried to break a ward rule so it's good because they're all watching each other and making sure that they're not breaking a rule but they maybe try it themselves but somebody else is watching them to break a ward rule as well so it's good. 


\title{
Perceptions of therapeutic principles within a therapeutic community
}

\begin{abstract}
Purpose: This study aimed to explore staff and service users' perceptions of therapeutic principles within a unique male high secure learning disability therapeutic community (LDTC).

Design/methodology/approach: A qualitative approach was adopted using deductive content analysis and inductive thematic analysis. Twelve participants took part in a semi-structured interview to explore their perceptions of Haigh's (2013) quintessence principles and any further additional therapeutic features in the environmentprinciples not captured by the theory.
\end{abstract}

Findings: All five quintessence principles were identified in the LDTC environment. Some limits to the principle of 'agency' were highlighted, with specific reference to difficulties implementing a flattened hierarchy in a forensic setting. Additional therapeutic featuresprinciples were identified including; security and risk, responsivity, and trust and-more physical freedom which appear to aid implementation of the quintessence principles.

Research limitations/implications: The study was performed within a single case study design. Therefore results remain specific to this LDTC. However, the finding of these principles in such a unique setting may indicate Haigh's (2013) quintessence principles are evident in other TC environments.

Originality/value: This is the first research paper that has attempted to test whether Haigh's (2013) quintessence principles are evident within a given therapeutic community. The research provides empirical evidence for the quintessence principles in a novel TC setting and suggests recommendations for future research.

Article classification: Research paper

Keywords: personality disorder, learning disability, therapeutic communities, forensic, secure. 


\section{Introduction}

The diagnosis of personality disorder (PD) within learning disability (LD) populations is prevalent within forensic settings (Blackburn et al., 2003) and associated with placements in higher security settings, serious and repeated offending and poorer long-term outcomes (Alexander et al., 2006; Torr, 2008). Consequently, effective treatments are important for individuals and wider society.

Democratic Therapeutic Communities (DTCs) have been commonly implemented in the treatment of personality disorder (PD) in non-LD populations (Rutter \& Tyrer, 2003), and recently LD populations (Taylor, Crowther \& Bryant, 2015). A DTC is defined as a 'living-learning situation' whereby, 'difficulties a member has experienced in relations with others outside are re-experienced and reenacted, with regular opportunities...to examine and learn from these difficulties' (Kennard, 2004: 296). DTCs are most usefully understood as a treatment modality (i.e. integrating a range of psychological and/or pharmacological approaches) as opposed to a specific treatment method itself (Kennard, 1998).

Literature on treatment of offenders with both an LD and PD remains limited, largely as a result of 'diagnostic overshadowing' and difficulty differentiating between symptoms of LD and PD leading to under diagnosis (Taylor \& Morrissey, 2012). Research on treatment for offenders with LD has indicated beneficial outcomes from adapted talking therapies, such as Cognitive Behavioural Therapy (CBT) and Dialectical Behaviour Therapy (DBT), with some case report evidence in existence for one to one psychodynamic therapy (Taylor \& Morrissey, 2012). Alternatively, growing evidence has been provided on the efficacy of DTC treatment in forensic LD populations (known as learning disability therapeutic communities, LDTC) in the form of reduced violence, personality pathology and interpersonal difficulties (Miles, 1969; Taylor, Crowther \& Bryant, 2015). 
The use of TCs within a learning disability population stems back to the 1940's where 'intentional communities' were first initiated (Kennard, 2004; Taylor, Crowther \& Bryant, 2015). These communities were developed specifically for an LD population, and most commonly known as the 'Camphill Communities'. Based within the community, the aim of the communities was to provide of sense of belonging for individuals often marginalised by wider society. This was accomplished via incorporation of values from traditions such as the 'Christian Mission' and 'Philanthropy' to provide a lifelong residential environment for individuals with LD, as opposed to operating as hospital or community based treatment programs (Haigh \& Lees, 2008). A number of core TC elements were adopted within community practice, including emphasis on equal status and the healing value of relationships. However, use of the psychodynamic model and analysis of social interaction was limited. Instead a particular focus was placed on practical work, as opposed to verbal exchange (Kennard, 2004).

Recently, the LDTC model has been introduced within a high secure setting at one of three high secure hospitals in the U.K. for males with a dual diagnosis of mild LD and PD, and produced equally successful results reduced PD pathology, relational difficulties and incidents of physical aggression (Morrissey \& Taylor, 2014). This is currently the only LDTC in existence within a high secure hospital.

Currently, treatment efficacy is generally evaluated against the favoured 'gold standard' form of research, such as RCTs (Haigh, 2005). However, a number of difficulties in generating 'gold standard' evidence for DTCs have been encountered; absence or reduced time of follow up, attrition, heterogeneity of outcome measures and patient population, participant selection and randomization, and establishing a suitable control group (see Capone, Schroder, Clarke \& Braham, 2016; Lees et al., 1999; Warren et al., 2003). The individualised nature of treatment has also limited measurement and standardization (Pearce \& Autrique, 2010). 
As such, the limited 'gold standard' evidence base for DTCs compared to other developing psychotherapy treatments for PD, such as Cognitive Behaviour Therapy (CBT) and Dialectical Behaviour Therapy (DBT) (Antisocial PD - NICE, 2009; Borderline PD - NICE, 2010) has prevented its inclusion within treatment recommendations (Pearce \& Autrique, 2010).

The number of issues arising from application of randomized controlled trial methodology suggests a post positivist approach to research design is incongruent with the complex nature of a DTC and consequently fails to capture its matrix of interrelated treatment components (Haigh, 2014). Some authors have therefore called for investigation of processes within DTCs to identify important treatment mechanisms that support therapeutic change (Aslan \& Yates, 2015; Magor-Blatch et al., 2014; Veale et al., 2014). Investigation of the lived experiences of those who comprise the community (service users and staff members) could be of particular importance in undertaking this research endeavour (Veale et al., 2014).

A number of theoretical schools - sociological, systemic and psychological, have informed development and functioning of therapeutic environments more generally (Haigh, 2015). For example, Rudolph Moos (1976) conducted extensive work into the personality of social environments and the processes and mechanisms within them that support change. Emphasis is placed on the physical structure of social environments. Increased physical space within a given setting is said to facilitate social and recreational activities, leading to increased cohesion amongst individuals and attraction of staff and residents with increased interpersonal skills who promote a sense of comfort and cohesion (Moos, 2012).

Practices central to TCs have also been understood in regard to psychoanalytic theories, such as Erikson's (1998) stages of psychosocial development and Mahler's (1985) separation-deindividuation theory of child development. Erikson's theory suggests a healthy developing individual is required to pass through eight stages from infancy to late adulthood. 
Passing through these stages begins at birth but unfold according to an individual's environmental and cultural upbringing.

Margaret Mahler (1985) suggested individuals navigate a 'separationindividuation' deficit from birth involving initial connection with one's surrounding environment before separating from attachment figures to develop a sense of self and identity over the first few years of life. The three stages (hatching, practicising and rapprochement) have been applied to understand individual experiences in group therapy (Fried, 1970).

While a number of theories have been specifically developed to delineate core features within DTCs, these accounts have adopted a more generic perspective. Rapoport (1960) identified four principles to describe the core elements of a TC environment leading to the development of therapeutic relationships via ethnographic research at the Henderson Hospital. Four core principles were identified to describe the main elements of a TC environment: Democratisation, Communalism, Permissiveness, and Reality confrontation (Rapoport, 1960). These principles were solely derived from the perspectives of staff members within the hospital (Debaere et al. 2016).

Haigh (2013) provided an update of the above principles, utilising his own clinical experiences and linking this to psychoanalytic and attachment theory. The clinical utility of Rapoport's (1960) themes was extended, connecting the above external experiences to psychological processes experienced by individuals. A developmental model was advocated, whereby individuals are thought to progress through five key conditions: 'attachment (belonging), containment, communication, inclusion, and agency' (Haigh, 2013, p. 6). In combination, these elements are hypothesised to provide the basis for emotional development leading to 'healthy personality formation' (Haigh, 2013, p. 6).

Neither Haigh's (2013) or Rapoport's (1960) theories has been subject to empirical verification in either secure or non-secure settings for individuals 


\section{with diagnoses of learning disabilities and personality disordercontemporary} niche TC settings, such as a male high secure LDTC.. Secure environments in particular come with their own set of challenges. As security and risk often remains on the forefront of the staff team's agenda, staff and patient relationships can become fractured as service users are restricted in a number of ways (Polden, 2010). For example, limited physical movement and established cultures discouraging contact between service users and staff (Polden, 2010) or being denied opportunities to address offence related factors on the basis of their disability (Taylor, 2010).

Within forensic TCs specifically, TC principles have been adapted to accommodate requirements of discipline and control (Rawlings, 1998). For example, the principle of agency is restricted so that service users can make decisions about the community without compromising the rules of the host institution. Individuals with an LD have been described to face further discriminatory experiences while in inpatient (NHS ENGLAND, 2015) and secure environments. Individuals with an LD can lack the capacity to manage or think about their feelings. Consequently, individuals' needs are often communicated behaviourally by 'acting out' (Gorman, 2015), which may further serve to reinforce the existing 'us and them' culture.

In sum, existing theory on TC processes has developed from a practitioner perspective, avoided subjection to empirical testing and maintained a generic focus despite the heterogeneous implementation of TCs in complex and specialist forensic settings. Although the single existing high secure LDTC has been evidenced to improve interpersonal difficulties and incidents of physical aggression, current research and theory is unable to imply whether suggested theoretical processes exist within this novel modified treatment setting. 
1. Explore both service user and staff members' perceptions of TC principles as outlined by Haigh (2013) and identify whether these are present in the environment of the LDTC within a high secure hospital.

Identify whether any further important principles exist within the social climate * of the LDTC that are not captured by current TC theory.
Formatted: Indent: Left: -0.25 ", Outline numbered + Level: $1+$ Numbering Style:

$1,2,3, \ldots+$ Start at: $1+$ Alignment: Left + Aligned at: 0" + Indent at: $0.25 "$

Formatted: No bullets or numbering

\section{Method}

\section{Design}

A single case study design was employed, with the 'case' being defined as the LDTC based at one of three high secure hospitals in the U.K. housing the high secure male learning disability population. A qualitative approach was employed within the case study to enable analysis of TC members' experience and perceptions of therapeutic principles in addition to identification of shared experiences. Data were collected via semi-structured interviews. The interview schedule included questions of experiences of Haigh's (2013) five TC principles, as well as general experiences within the EDTC. Questions were adapted for service users to ensure language remained accessible.

The semi-structured interview started with some specific questions aboutFormatted: Space After: 0 pt Haigh's five quintessence principles to facilitate a discussion on areas detailed in existing theory. TC principles are notoriously difficult to capture as they refer to pre-verbal experiences associated with emotionally lived experience (R. Haigh, personal communication, 2015). Interview questions were therefore refined via discussions with clinicians who had previously 
worked or resided in TCs and were consequently familiar with the philosophy and experiences within such establishments.

Thereafter, a number of broader questions were asked to elicit participants' views on any additional experiences in the LDTC that remain uncounted for by current theory. Questions used enabled service users to use their own language in describing other alternative experiences in the TC. For example, 'If your TC were an animal, what would it look like?' When conducting the interview with service users, a number of additional prompts were used. After initially presenting the first open question, follow up questions (in an either/or format) were used to support the individual in answering the question, if required, without leading them. These questions were implemented to support individuals who find abstract concepts difficult to comprehend and require questions to be more concrete in nature to provide a response (Nind, 2008).

Pictures were used to support understanding and prompts in an either/or format were also provided when required for questions involving abstract concepts.

\section{Ethics}

The study was approved by Lincoln University ethics committee and Leicester Central NHS Research Ethics Committee.

\section{Recruitment and data collection}

Participants (staff members and service users) were recruited from a male LDTC at one of three high secure hospitals in the U.K. All TC members were invited and therefore no specific sampling strategy was used.

The inclusion criteria for staff member participation were: permanent employment within the LDTC for a minimum of three years to ensure individuals harboured a thorough understanding of the processes of this

Formatted: Space After: $0 \mathrm{pt}$

Formatted: Body Text 3, Left

Formatted: Right: 0.25 
complex treatment modality. Similarly, all staff members were required to be able to communicate and understand verbal/written English to facilitate full engagement in the interview process. Those who did not meet the inclusion criteria above were excluded from the research, although everyone who volunteered to participate met inclusion criteria.

-Ideally, equal numbers of service users and staff members were aimed to be interviewed within the study. Before commencing the interview, all participants reviewed the information sheet and had the opportunity to ask questions prior to signing a consent form. Interviews were completed by the first author and lasted between 59-103 minutes.

\section{Participants}

Twelve participants took part in the study (six staff members and six service users). Out of the 12 service users invited to take part in the study, six (50\%) consented to take part. These individuals did not provide any reasons as to why they did not wish to engage with the research and due to lack of consent it was not possible to explore demographic information and determine whether these individuals differed in any way to those who participated.

Twenty out of 40 members of the staff team remained on permanent night shifts and it was therefore not possible to recruit these individuals in to the research. Out of the remaining 240 staff team members, seven (18\%) staff members were eligible to partake in the study based on permanently working on the LDTC and having equal to or more than three years of experience in working in the setting. and- $\underline{S}$ six of the seven eligiblese individuals consented to partake in the study (one TC Manager, two Nurses and three Healthcare Assistants). Again, the individual who declined to participate did not provide any reasoning for their decision not to participate.

All service user participants were male. Two staff participants were female and four were male. All service users' IQ scores resided within the mild range for learning disabilities. Table 1 details further participant demographics of those who took part in the study in the LDTC. 


\section{INSERT Table 1: Participants demographics - means and ranges}

\section{Analysis}

The interviews were recorded with a digital Dictaphone and transcribed verbatim. The data was then subjected to deductive content analysis (Mayring, 2000). Inductive thematic analysis was performed on remaining data. This followed a six-step process described by Braun \& Clarke (2006). Saliency analysis (an enhancement of thematic analysis) was then utilised to justify the selection of themes and ensure identification of codes that did not recur although remained important to the research questions posed (Buetow, 2010) (see extended analysis).

\section{Trustworthiness}

To ensure trustworthiness, the following four criteria were adhered to throughout the study; credibility, transferability, dependability and confirmability (Guba, 1981; Shenton, 2004). To increase credibility and transferability of analysis and results, supervision was used regularly. In addition, a wide range of informants were utilised in the form of staff and service users to verify individual view points and experiences against others and thus gain a more stable view of reality. 
Further, to ensure credibility of the deductive coding template, a colleague and the first author independently coded two transcripts (one staff and one service user transcript) to improve reliability of ratings provided for the qualitative responses.

To establish inter-rater agreement, coded staff and service user transcripts were subject to statistical analysis in order to account for the possibility of chance agreement (Weber, 1990). The averaged Kappa coefficient across all five categories coded for was 0.79 for the service user transcript and 0.80 for the staff transcript, both indicating 'substantial agreement' (Viera \& Garrett, 2005). The final set of coded data represents agreed ratings.

To address dependability, an audit trail was completed comprising of transcripts and annotations. Confirmability of findings was increased by engaging in a reflective process throughout the research, in the form of a research diary.

\section{Results}

\section{Are Haigh's (2013) quintessential elements of a therapeutic environment} present in the environment of the LDTC within a high secure hospital according to service user and staff members' perceptions?

Overall, staff and patient responses were consistent with Haigh's quintessence principles of therapeutic environments. All participants (staff and service users) reported to experience all five of the quintessence principles in the LDTC, albeit to varying degrees (please see Table 2 below).

\section{INSERT Table 2: Frequencies of categories endorsed by participants} based on Haigh's quintessence principles 
For example quotes for each of the following categories, please see Appendix $A$ and also extended results section within the extended paper.

\section{Attachment}

The first category posed by Haigh (2013) required for 'secondary emotional development' relates to attachment. Both service users and staff described experiences of attachment within the LDTC (patient - 17; staff - 29). Five out of six service users referred to experiences of belonging and feeling valued. Similar experiences were described by all participating staff. Comments pertaining to attachment were made to a lesser extent compared to participants' experience of other TC principles. Further, staff members mentioned attachment more frequently (29) than service users (17).

\section{Containment}

The second category proposed by Haigh (2013) relates to containment (opportunity to express emotions and gain valued support, awareness of boundaries). Service users mainly mentioned valued experiences of support from both peers and staff. While staff frequently mentioned experience of valued support, they also commonly referred to the importance of giving people time and space to display and experience emotions without immediate staff intervention. All service users (6/6) and staff (6/6) mentioned containment and described experiencing containment to a similar degree in the LDTC overall (service users -51 ; staff -58 ).

\section{Communication}

Communication was the third principle put forward by Haigh (2013) in his understanding of what constitutes a therapeutic environment. Service users mentioned experiences of enquiry, commentary, and questioning. References were also made to feeling safe in the fact the community will accept what they have to say. Similarly, staff mentioned the above features of communication. While all service users (6/6) and staff (6/6) demonstrated experience of 
communication, this was mentioned slightly more by staff (67) compared to service users (53).

\section{Involvement and Inclusion}

The fourth principle refers to involvement and inclusion. Service users described involvement and inclusion as mainly promoted via peer pressure and rules and procedures. Staff members also regularly mentioned the above features, in addition to staff intervention to promote involvement and inclusion. While all participating service users (6/6) and staff (6/6) experienced the concept within the LDTC, staff members described instances of involvement and inclusion (63) more often than service users (35).

\section{Agency}

The final principle posited by Haigh (2013) relates to agency. Service users mainly referred to experiences of agency involving shared responsibility within specified limits. Staff also frequently referred to experiences of shared responsibility, in addition to peers policing each other. While all service users $(6 / 6)$ and staff $(6 / 6)$ described experiencing the concept of agency within the LDTC, this was mentioned more frequently by staff $(78)$ than service users (46).

\section{Do any further important principles exist within the social climate of the} LDTC that are not captured by current TC theory? 
The results are presented with reference to a thematic map (see Figure 1), which outlines a number of themes related to additional principles in the LDTC environment along with their prevalence/importance. The main themes identified were labelled Security and Risk, Trust, More Physical Freedom and Responsivity. The themes and their respective subthemes are discussed below. The paper focuses specifically on those themes that were recurrent and important to participants as to be considered a TC principle, concepts need to be generaliseable to the LDTC as a whole. Other important but not recurrent themes included; 'moving on', 'being reflective', 'staff fit with the LDTC'.

\section{INSERT - Figure I: Thematic map}

\section{Security \& Risk}

While not mentioned as frequently by service users $(2 / 6)$ security and risk was discussed more regularly by staff (5/6). When talking about security and risk, participants highlighted two subthemes, which relate to management of security and risk within the LDTC and its high secure status: 'observations' and 'searches'.

Observations 
Two service users highlighted being placed on clinical observations occurred from time to time on the LDTC and that it can be 'hard getting back' to where you were before:

[...] And getting back up is the hardest part of doing it because you know if they like put you on sight and sound or something like that you know you've got to be good to get off that sight and sound or they can put you on watch where you go in your room at night-time they either leave your bedroom door open special watch or your hatch open, you've got to be spot on to have it shut, you've got to be alright that's quite hard getting back up over that.

One patient went on to describe the restrictions experienced when placed on high level observations in more detail impacting on their opportunity to engage in off ward activities:

[...] Yeah or not taking any medication with me if I don't take my medication I'll have my keys took off me, me bedroom locked, day-room bound, can't move, can't do anything, got to hand my keys into them so I mean I'm one of the worst people on the ward to get hit if they refuse anything because I'll get everything stopped and I can't afford that nowadays and I hate staying on the ward.

A further participant went on to explain how lower level observations, such as overseeing interactions between visitors and service users, are more flexible and remain dependent on visitor preference; something that does not occur on other wards:

[...] Like for example with you being in this room now like you said you could have had a member of staff in with us if you wanted, if we wanted one, on other wards it would have been if we'd wanted it or not a member of staff would be sitting in with you, a member of staff would be in the corner near the door and we'd be here doing our talk and the member of staff would be observing at all times but since we're on a therapeutic community the member of staff's only like even not that far away, he's on the bench watching us and he's watching us from a distance but on other 
wards a member of staff would be in the room while we're doing this oneto-one or this session what you're doing for your research.

\section{Searches}

Five staff members discussed the use of patient searches as part of high secure hospital policy:

[...] Umm, I mean obviously working within high secure there are policies there so in a sense, at times, that's where the decisions come from so for example if you're going off ward then you have to have a rub down search, that's in the policy so that's not staff making that decision that's working within the policy.

One member of staff went on to describe the variety of searches service users experience on the LDTC and service users' acceptance of such procedures on the LDTC:

[...] I mean there are, there are certain things that obvious security things that, you know, there's no question about they will have a room search done once a month, they'll have a couple of locker searches done, they will be subject to rubdown searches on their way out, there's the obvious things like that and they all accept that, they know that's out of our hands we have to do that, it's for their safety and our safety, they'll accept that.

\section{Trust}

This theme ties in with security and risk, in that flexibility with security conditions remains dependent on trust between staff and service users. The importance of 'trust' within the LDTC was mentioned frequently by both service users (4/6) and staff (5/6). Participants who spoke about trust in the LDTC highlighted two subthemes relating to how trust is developed between service users and staff along with its importance and influence on care provision: 'learning to trust' and 'staff spend more time'. 
process in order to be able to communicate openly with each other. For example, one patient stated:

[...] Why because you've got to learn to trust them to be able to talk to them about problems and childhood and all sorts and all your past history and everything.

This sentiment was further echoed by staff members:

\section{[...] Again I think it builds up their trust that maybe individuals have found} very hard to have in the past, maybe a lot of their history hasn't allowed certain individuals to trust people and they find it hard to trust people maybe on here it's just sort of I say twenty-four hours it can happen say that trust just sort of gets another sort of brick added to it on a daily basis then cements that relationship, which then allows more openness, again the relationships build and build and develop, again that's the model patient to patient, patient to staff, staff to patient.

One staff member described trust between staff and service users to develop via patients observing staff members support other service users with their problems:

[...] Things were being brought up and things were getting sorted pretty quickly and they were starting to see that, oh if I've got a problem my problem will be sorted pretty quickly and I think that's what brought the trust together if you like ... and I think that's how the trust just built itself over the years we've been here.

\section{Staff spend more time}

An additional method through which trust is built may be through time. Five participants (1/6 service users $4 / 6$ staff) expressed valuing spending time with each other on the LDTC. From one service user's perspective, they felt this showed staff 'care for patients': 
[...] But on the TC you've got staff who just spend time being around patients, care for patients instead of being somewhere else, i.e. like office or kitchen.

Similarly, some staff members linked engaging in 'simple' activities on a regular basis with the development of staff and patient relationships:

[...] We spend a lot of time doing things, simple things together, it may only be sitting playing cards, it may be sitting playing Monopoly but we spend a lot of time with our patients and that makes relationships far easier.

[...] there's no like budget to buy staff meals and all that type of thing so staff do sit down like on an individual basis and have their own sort of food with patients.

\section{More Physical freedom}

'More physical freedom' shares links with security and risk, and trust, as participants portrayed providing service users with freedom as remaining dependent on trust held between staff and patients and ultimately overall limits set by the hospital in order to manage security and risk. Both service users (5/6) and staff (4/6) frequently spoke of how much they valued the extra physical freedom afforded to patients within the TC.

From a service user perspective, one individual stated:

[...] it's more laid back than the other wards, on the other wards if you've been in the dining room and you get up and go to your room and then you've got to go to bed at a certain time. On here you don't go to your room until quarter to nine and then after you've done your groups, like when you do your group on a Friday afternoon then there's more time to do what you want to do but you can't do that on other wards, you can come in here, go on the Wii, have a cup of tea whenever you want, you can't do that on any other wards.

A number of staff members highlighted how patients do not need to ask permission to move around in their environment: 
[...] Basically what l've seen in the past they're told to sit down, they have to ask to get up, to go to the toilet and all that, whereas on here there's a lot more sort of freedom, they can go to their rooms when they want if they've not got activities and things.

One patient went on to describe how the physical freedom afforded to patients enables staff and patients to sit together outside of meetings and 'have a laugh':

[...] But when you're on the ward after the meetings you can still sit and have a laugh and a joke with everybody, it's not like some wards where it's all strict, day-room bound and all doors locked off, all doors are open.

\section{Responsivity}

The importance of the final theme, responsivity, was also highlighted to a similar extent by both patients (4/6) and staff (5/6). Three subthemes were identified based on three main ways TC members described tailoring their approach in responding to situations within the LDTC: 'knowing your patient', 'giving people time and space' and 'making allowances'.

\section{Knowing your patient}

This theme ties in with the prior theme of trust, particularly in regard to 'staff spending more time' with patients and 'learning to trust'. Four out of six staff members reflected on how time spent informed knowledge gained about patients and helps to build relationships between staff and patients:

[...] I like that all staff have an in depth knowledge of patients, nursing assistants and qualified staff, an in depth knowledge. The relationships on here that have built up because of the knowledge that staff have got and the experiences that the staff have had with the patients.

[...] But obviously the TC, everybody knows everybody, well staff know, staff know the patients, they know their problems and that's the difference in working anywhere else. 
A number of staff members expressed how knowledge held by staff regarding patients along with the relationships built have a direct impact on care provided by informing the way staff approach patients. For example, one staff member described noticing a change in a patient's body language, and by having some knowledge of the patient they were able to act on this and offer support:

[...] But you know you can tell by body language basically that a certain individual, you know something's not right...having worked with those patients for so many years, you know when something's not right and you can approach a patient and say look, you know, what's happening.

\section{Giving people time and space}

A number of patients $(3 / 6)$ discussed the importance of providing people with time and space on the TC when tailoring their approach to individuals. From a patient perspective, three individuals expressed how much they valued the time and space given by staff, and particularly peers, to talk when they are ready, which had not been provided to them on other wards:

[...] Yeah but some days you might find it hard, like on this ward you have to talk about what's troubling you straight away and then you can talk or we'll give you a bit of space and time and then you can talk when you're ready, not there and then but on other wards probably get told no you need to talk it now.

[...] It all depends what kind of mood you're in, if you're not in a good mood you want to be alone then, patients respect that and give you a bit of space.

One patient went on to express how fellow patients look out for each other and warn others to give people space when they need it:

[...] that person who's the same group as you asks how you're feeling and you say to that person that you're not feeling alright, then that person gives you a bit of space and if that person sees someone else trying to keep asking then that person who's asking are you feeling alright and that person no you're not, then that person tells the other person just to leave you alone.

Formatted: Right: 0.25 
While providing people with time and space to choose when to share their difficulties with others, patients continue to monitor other peers' wellbeing when they are aware they are 'not alright':

[...] But even sometimes when you know they're not alright they'll still say yeah which is frustrating when you know you want to help but obviously if they don't want it at the time you've just got to wait and just keep an eye on them to make sure they're alright.

\section{Making allowances}

A further way of adapting methods of responding to others was highlighted in the form of 'making allowances'. More staff (3/6) than patients $(2 / 6)$ discussed the use of making allowances for others depending on the situation. All three staff members discussed remaining 'sensitive' to 'mitigating circumstances'. For example:

\section{[...] Sometimes there can be mitigating circumstances with certain things and then obviously we'll be sensitive to that and probably won't follow certain things through if that's the case.}

[...] The only time where we sort of say to them, you know, fair enough, you'd ask them if they're not very well or they've had bad news or whatever, then fair enough but if they're just basically like I'm not going [to the community meeting] then there's consequences for them.

Two patients went on to confirm this based on their own experiences. One patient described the following scenario:

[...] Like tonight we can go mixing on the other side, Thursday you don't mix but if I'd had a bad phone call from my family I just see a member of staff and say can I have permission to go and speak to someone over there so I can get a bit of support because this has happened nine times out of ten they'll say yeah go on, just let the staff know, that's what you get. 


\section{Existing TC principles and additional principles - A summary}

While all TC principles were confirmed by staff and service users, albeit to varying degrees, a number of additional features in the environmentprinciples were also identified via inductive analysis.

Security and risk can be considered linked to containment in that conditions of security comprise some of the boundaries via which members are aware of what behaviour is and is not permitted in the LDTC.

However, due to the high security status of the hospital, these boundaries are qualitatively different to that which may be found in, for example, a community day TC or even low/medium secure TC and therefore deserve individual consideration.

The theme of trust is clearly linked to concept of s of attachmentcontainment in terms of However, rather than pertaining specifically to members feeling valued by others in the TC and experiencing a sense of belonging, trust appears to relate more specifically to the confidence members have that $\mathrm{TC}$ members exadequate support in general will be provided when they require it, allowing them to feel safeperiencing a degree of emotional safety to a degree where they are able toenabling them to communicate their difficulties to TC members to access support. Features that support learning to trustmembers developing a sense of emotional safety appear related to development of attachments between staff and patients, facilitated by staff spending more time with service users and patients seeing other members' problems become solved through process of involvement and inclusion. 'More physical freedom' harbours links with communication, in that physical freedom facilitates further opportunities for informal conversations to take place.

Finally, the theme of responsivity is linked to containment, communication, involvement and inclusion and agency, as depending on the situation at hand this may involve applying one or a combination of 
these principles. However, prior to this, the individual is required to consider the service users' current presentation/circumstances and consider how best to approach and/or support them based on this, which may involve flexibility in application of all TC principles.

For example, giving people time to feel comfortable to communicate with others and/or receive support alongside forgoing community meetings where appropriate and necessary.

\section{Discussion}

\section{Existing TC principles}

Overall, the majority of service users and staff confirmed Haigh's TC pPrinciples as evident in the LDTC environment. However, the frequency with which TC principles were mentioned by staff and service users differed considerably. Staff in particular emphasised agency, involvement and inclusion over service users. It is possible staff discussed these concepts more frequently due to their distinct departure from previous ways of working in forensic environments, particularly with individuals diagnosed with an LD. For example, the principle of involvement and inclusion lies in direct contrast with usual approaches in secure environments where individuals with an LD are denied opportunities to address offence related factors on the sole basis of their disability (Taylor, 2010).

As the majority of participating service users confirmed the five TC principles as evident in the LDTC, the reduced frequency with which they were discussed, when compared to staff accounts, may relate to individuals' difficulties in describing the concepts. Difficulty in communicating concepts may explain why the principle of containment, for example, was described with equal frequency by service users and staff.

-Containment is easier to describe as it is constantly visible on the unit in others behaviour and therefore more tangible in nature. The concept of attachment on the other hand is a less tangible/pre-verbal experience (Haigh, 
2013). Consequently, this principle may prove more difficult for individuals in general to describe and discuss, particularly for those with an LD.

\section{Additional therapeuticTC principles}

Twohree additional features in the environmentprinciples were identified in analysis, which were both recurrent and considered important by the majority of staff and service users; Trust,More physical freedom, and Responsivity.

While these features may not primarily contribute to the experience of secondary emotional development outlined by Haigh's (2013) five quintessence principlesAll__of the above themes could be considered to belongremain important considerations withinto therapeutic environments in light of their role in facilitating enactment of TC principles within secure environments, such as the LDTC.

More physical freedom

More physical freedom plays an important role in facilitating existing TC principles. Typically physical freedom is constrained in high secure settings (Polden, 2010).

While Haigh's (2013) principles focus on the emotional culture of an environment, practical elements, such as increased physical freedom, are required in order for service users to have opportunities to engage in therapeutic interactions with peers involving communication, expressing emotions and experience these being contained by peers/staff, in addition to being able to start practicing agency over their own behaviour in simple ways such as choosing where to locate themselves. The importance of physical freedom has previously been highlighted by Moos (2012). Similarly, Moos (2012) suggested physical space within a given setting could facilitate increased social and recreational activities, leading to increased cohesion amongst individuals. 


\section{Responsivity}

Participants also considered responsivity from staff and peers important within the LDTC. By spending time with service users, staff gain further knowledge about individuals and are able to adapt how they respond in terms of bearing in mind individual circumstances when applying TC principles and considering the nature of support required in the present moment. As a result, service users felt their individual needs were more adequately gauged and responded to by the team.

Individuals' needs within group therapy have commonly been understood in relation to attachment literature (Fried, 1970; Mahler, 1968). Mahler (1985) suggested individuals navigate a 'separation-individuation' deficit involving 
initial connection with one's surrounding environment before separating from attachment figures to develop a sense of self and identity.

The three stages (hatching, practicing and rapprochement) require careful navigation and each individual will travel along their own trajectory at their own pace. As some service users interviewed had resided at the TC for 4.5-5 years, they may have passed through to the rapprochement stage (Mahler, 1985), where they are focused on developing their own identity/separate from group placing more value on agency. Consequently, these individuals' needs may differ from those who have resided at the TC for a shorter duration. Flexible application of TC principles may therefore be important in order to remain responsive to TC members and their current needs based on the developmental stage they have reached during their time on the LDTC.

However, responsivity and the subthemes within it were mainly discussed in relation to staff being responsive to patients versus patients being responsive to staff. Consequently, descriptions of how care is provided and who provides care (largely staff for patients) may have implications for how far the agency principle can be enacted in a high secure environment such as the LDTC, with respect to a flattened hierarchy. Recommendations for exploring this issue further are made in the 'research implications' section.

\section{Security and Risk}

The theme of security and risk was mainly emphasised by staff and slightly smaller than other three themes. The theme of Security and Risk is largely related to the nature of the LDTC running in a high secure setting (Polden, 2010) rather than being an additional therapeutic principle. However, this remains an important area for consideration in regard to how and whether existing TC principles can be employed around management of security and risk. 
Trust was also considered important by TC members in order to allow people to feel safe that communicating their problems to staff and patients will lead to support and containment. Staff also learn to trust patients, for example, by providing them with more physical freedom. While trust can be understood in relation to Haigh's (2013) conceptualisation of the containment principle and TC members experiencing a sense of emotional safety, Irust is a particularly pertinent concept for consideration in secure settings where relationships between staff and service users are often fractured (Polden, 2010).

The importance of trust has been reflected in Erikson's (1998) psychosocial theory of development, specifically the Hope: trust versus mistrust stage (0-1 years).

As a result of sufficient attachment experiences (being nurtured and loved), individuals develop a sense of trust in others. Without this, the infant develops a high level of mistrust, causing them to become withdrawn in later life.

Sufficient exposure to the attachment principle in TC environments (engendering a sense of belonging and feeling valued) and involvement and inclusion (seeing others needs consistently met by staff as caregivers) may create a sense of trust in others, encouraging communication and providing opportunities for containment.

\section{Clinical implications:}

Does the LDTC fit with a high secure system?

While Haigh's (2013) TC principles are evident in the novel environment of the male LDTC, it is evident that the high secure nature of the LDTC environment modifies and influences their implementation, particularly in regard to agency.

This is not necessarily surprising as existing literature has highlighted difficulties in maintaining therapeutic program integrity in TCs based in secure host institutions, with particular reference to limits imposed on agency (Rawlings, 1998). 
While there are specified limits to the amount of agency service users can experience, there is still evidence of its existence, particularly with regard to service users being empowered to have a say in how their community is run (see Appendix A).

Implementation of TC principles within a high secure setting appears aided by additional featuresprinciples of trust, responsivity and more physical freedom. While trust is captured within Haigh's (2013) principle of containment, it is particularly important emphasis is placed on developing a sense of trust between staff and service users via purposeful effort of the community engendering an experience of emotional safety for TC members.

Relationships in forensic settings between staff and service user groups are often characterised by hostility and mistrust (Polden, 2010).

In addition, individuals with an LD can lack the capacity to manage or think about their feelings and therefore communicate their needs behaviourally by 'acting out' in an attempt to rid themselves of their feelings (Gorman, 2015). However, with increased physical freedom, TC principles of involvement and inclusion and open communication can be fully enacted.

Strong therapeutic relationships are developed that are able to tolerate high levels of aggression and risk within LD/PD forensic populations (Alexander et al., 2006; Torr, 2008). These relationships provide a platform for trust and a sense of emotional safety to grow, enabling staff to respond flexibly and effectively to each individual's needs.

The future of the LDTC

Compassionate and nurturing relationships are of particular importance to forensic LD populations in light of frequent experiences of historically being deprived of having the opportunity to be responsible over their own care (Taylor, 2010). Such issues lie at the centre of Transforming Care Paper (NHS England, 2015). 
The principles inherent in the LDTC environment could provide a pathway for forensic populations with a dual diagnosis of PD and LD in conditions of high, medium, low security and step down facilities.

This pathway could help to both safeguard individuals from abuse via communication and involvement and inclusion, and emphasise individual agency, while supporting service users to apply skills from the TC to external/'real life' environments, as risk reduces.

Limitations and research implications:

A strength of the study is that it provided an in-depth exploration of TC principles within the only existing male LDTC in a high secure hospital, and recommendations for the direction of future research in this area. The study was performed within a single case study design and therefore results remain specific to this LDTC.

However, the finding of TC principles in such a unique and high secure setting may indicate Haigh's (2013) TC principles are evident in other, less constrained, LDTC environments. As there is no existing research exploring TC principles in LDTCs in lower conditions of security or non-secure settings, this highlights an area for future research to explore.

The study should also be interpreted with reference to its limitations. Participants who left the LDTC prior to treatment completion could not be invited to take part in the study due to no longer residing at the hospital. This may have excluded alternative perspectives on TC principles inherent within the LDTC.

The study also neglected to explore reciprocity of TC principles more explicitly with staff members. For example, within the interview schedule the research could have enquired further in regard to staff experiences of communication in terms of how open they are with service users and information they choose to disclose or not disclose. Consequently, the research provides limited 
information on whether and how TC principles work on a two-way basis between service users and staff members. To explore this further future research could take the form of naturalistic observations via an ethnographic study.

Being able to observe processes within the LDTC as they unfold may help to investigate how far TC principles, such as agency, and other features of general care such as 'responsivity' are enacted within a high secure environment and determine how much these features apply to staff as well as service users.

While the study identified existing and additional TC principles inherent in the LDTC, it did not specifically consider how these might inform outcomes within the LDTC. One method of linking principles and outcomes may be to evaluate how important TC principles are to individuals. The importance of TC principles to staff and service users may have implications for the development and maintenance of individual and group alliances between staff and service users. This should be thecould also provide a focus foref future research in order clinically inform future LDTC environments in high secure settings.

\section{Future research could develop a process-based measure made up of} statements representing core processes in the LDTC agreed to exist by the community. Statements could be derived from qualitative data from this study detailing therapeutic principles inherent in the LDTC environment. Participants could then be asked to individually sort the statements in terms of importance, for example utilising a $Q$ sort procedure. Each individual's $Q$ sort could then be subject to quantitative analysis to identify mutually agreed important therapeutic elements of the social environment as identified by the community. 


\section{Conclusions:}

While the high secure nature of the LDTC appears to modify and influence application of TC principles to the environment, findings of the study highlighted confirmation of existing TC principles in this niche and novel environment by staff and service users. Staff and service users also confirmed a number of additional features within the LDTC environment.

Wthathile these features are not primarily linked to Haigh's (2013) principles they -help to facilitate implementation of existing TC principles that provide an experience of secondary emotional development within inform - athe therapeutic environment in conditions of high security; - Trust, Responsivity, More physical freedom. While the theme of trust is captured within Haigh's (2013) conceptualisation of the principle of containment, it is argued this principle requires particular attention within secure settings in order to develop a sense of emotional safety within an LDTC. It seems prudent these additional elementsprinciples are emphasised and nurtured in order for the LDTC to continue to thrive in a high secure setting, and potentially conditions of lower security in the future. 


\section{References}

Alexander, R.T., Crouch, K., Halstead, S. and Piachaud, J. (2006), "Longterm outcome from a medium secure service for people with intellectual disability", Journal of Intellectual Disability Research, Vol. 50 No. 4, pp. 305-315.

Aslan, L. and Yates, R. (2015), "Exploring the "black-box" of therapeutic community (TC) methodology and the subjective experiences of residents within TC structures", Therapeutic Communities, Vol. 36 No.

2.

Barker, C., Pistrang, N. and Elliot, R. (2002). Research methods in clinical psychology, $2^{\text {nd }}$ ed.. London: John Wiley \& Sons. 
Baumeister, R. and Bushman, B. (2003). Emotions and aggressiveness. In W. Heitmeyer and J. Hagan (Eds), Internaitonal handbook of violence research (pp. 479-493). Dordrecht, The Netherlands: Kluwer.

Blackburn, R., Logan, C., Donnelly, J., and Renwick, S. (2003), "Personality disor- der, psychopathy, and other mental disorders: Comorbidity among men- tally disordered offenders in English and Scottish high security hospitals", Journal of Forensic Psychiatry, Vol. 14, pp. 111137.

Birtchnell, J, Shuker, R., Newberry, M. and Duggan, C. (2009), "An assessment of change in negative relating in two male forensic therapy samples using the Person's Relating to Other's Questionnaire (PROQ)", Journal of Forensic Psychiatry and Psychology, Vol. 20 No. 3, pp. 1-21.

Braun, V. and Clarke, V. (2006), "Using thematic analysis in psychology", Qualitative Research in Psychology, vol. 3, no. 2, pp. 77-101.

Bumby, K. (2000). Empathy inhibition, intimacy deficits and attachment difficulties in sex offenders. In: D. R. Laws, S. M. Hudson and T. Ward (Eds), Remaking relapse prevention with sex offenders: A sourcebook. Thousand Oaks: Sage.

Capone, G., Schroder, T., Clarke, S. and Braham, L. (2016), "Outcomes of therapeutic community treatment for personality disorder", International Journal of Therapeutic Communities, Vol. 32 No. 2, pp.84-100.

Debaere, V., Vanheule, S., Van Roy, K., Meganck, R., Inslegers, R., and Mol, M. (2016), "Changing encounters with the Other: A focus group study on the process of change in a Therapeutic Community", Psychoanalytic Psychology, Vol. 33 No. 3, pp. 406-419.

Erikson, E. (1998). The Life Cycle Completed (Extended Version). New York: Norton \& Company. 
Fried, E. (1970). Individuation through group psychotherapy, International Journal of Group Psychotherapy, Vol. 20 No. 4, pp. 450-459.

Gilbert, P. (2005). Compassion and cruelty: A biopsychosocial approach. In: P. Gilbert (Ed). Compassion: conceptualisations, research and use in psychotherapy (pp. 263-326). London: Routledge.

Haigh, R. (2005), "The Trouble with Modernisation: We Need Better Relationships, Not Policies and Procedures", Mental Health Review Journal, Vol. 10 No. 3, pp. 3-7.

Haigh, R. (2013), "The quintessence of a therapeutic environment", Therapeutic Communities, Vol. 34, No. 1, pp. 6-15.

Haigh, R. (2014), "What is happening in the world of therapeutic communities?", European Journal of Psychotherapy and Counselling, Vol. 16, No. 1, pp. 74-76.

\section{Haigh, R. \& Lees, J. (2008), "Fusion TCs: Divergent histories, converging} challenges", Therapeutic Communities, Vol. 29, pp. 347-374.

Formatted: Font: Italic

Hanson, R. K. (1997). Invoking sympathy: assessment and treatment of empathy deficits among sexual offenders. In: B. K. Schwartz and H. R. Cellini (Eds). The sex offender: New insights, treatment innovations, and legal developments. Vol II Kingston. Civic Research Institute.

Kear-Colwell, J. and Pollock, P. (1997), "Motivation or confrontation: Which approach to the child sex offender?" Criminal Justice and Behaviour, Vol. 24, pp. 20-33.

Kennard, D. (1998), "Therapeutic communities are back - and there is something a little different about them". Therapeutic Communities: The International Journal for Therapeutic and Supportive Organisations, Vol. 19, pp. 323-329.

Formatted: Right: 0.25 
Kennard, D. (2004), "The therapeutic community as an adaptable treatment modality across different settings", The Psychiatric quarterly, Vol. 75 No. 3, pp. 295.

Lees, J., Manning, N. and Rawlings, B. (1999), Therapeutic community effectiveness: a systematic international review of therapeutic community treatment for people with personality disorders and mentally disordered offenders, University of York.

Lees, J., Manning, N. and Rawlings, B. (2004), "A culture of enquiry: research evidence and the therapeutic community", The Psychiatric quarterly, Vol. 75 No. 3, pp. 279.

Lewis, M. (1995), "Self-conscious emotions", American Scientist, Vol. 83, pp. 68-78.

Magor-Blatch, L., Bhullar, N., Thomson, B. and Thorsteinsson, E. (2014), "A systematic review of studies examining effectiveness of therapeutic communities", Therapeutic Communities, Vol. 35 No. 4, pp. 168-184.

Mahler, M. (1968). On Human Symbiosis and the Vicissitudes of Individuation: Infantile Psychosis, Vol. 1. New York: International Universities Press.

Mayring, P. (2000), "Qualitative content analysis", Forum: Qualitative Social Research, vol. 1, no. 2. Retrieved August 28, 2016, from http://217.160.35.246/fqs-texte/2-00/2-00mayring-e.pdf.

Miles, A. (1969), "Changes in the attitudes to authority of patients with behaviour disorders in a therapeutic community", The British journal of psychiatry : the journal of mental science, Vol. 115 No. 526, pp. 1049.

Moos, R. (2012), "Evaluating the environment of residential care settings", The International Journal of Therapeutic Communities, Vol. 33 No. 2/3, pp. $76-85$.

Morrissey, C. and Taylor, J. (2014), "Changes in Personality Disorder Traits 
Following 2 Years of Treatment in a Secure Therapeutic Community Milieu", Journal of Mental Health Research in Intellectual Disabilities, Vol. 7 No. 4 , pp. 323-336.

National Institute for Health and Clinical Excellence (2009). "Antisocial personality disorder: Treatment, management and prevention", available at: https://www.nice.org.uk/guidance/cg77 (accessed 27 September 2015).

National Institute for Health and Clinical Excellence (2010), "Borderline personality disorder: Treatment and management", available at: https://www.nice.org.uk/guidance/cg78 (accessed 27 September 2015).

Newton, M. (2010). Changes in Prison Offending among Residents of a Prison-Based Therapeutic Community. In: Shuker, R. \& Sullivan, E. (Eds.). Grendon and the Emergence of Forensic Therapeutic Communities: Developments in research and practice. Chichester, U.K.: John Wiley \& Sons, pp. 281-291.

NHS England (2015). Transforming care for people with learning disabilities Next steps. Available at: https://www.england.nhs.uk/wpcontent/uploads/2015/01/transform-care-nxt-stps.pdf (accessed 2/12/2016)

Nind, M. (2008). Conducting qualitative research with people with learning, communication and other disabilities: methodological challenges.

Available at: http://eprints.ncrm.ac.uk/491/1/MethodsReviewPaperNCRM-012.pdf (accessed 7/5/2017). 
therapeutic community approaches", Therapeutic Communities, Vol.31 No.4, pp. 338-355.

Polden, J. (2010), Behind locked doors: An exploration of therapeutic processes within a prison therapeutic community, British Journal of Psychotherapy, Vol. 26 No. 4, pp. 502-521.

Rapoport, R.N. (1960), Community as doctor : new perspectives on a therapeutic community / Robert N. Rapoport with the collaboration of Rhona Rapoport and Irving Rosow ; introduction by Maxwell Jones, London : Tavistock Publications.

Rawlings, B. (1998), "Research on therapeutic communities in prisons: A review of the literature", available at: http://www.dldocs.stir.ac.uk/documents/rawlings.pdf (accessed 27 September 2015).

Rutter, D. and Tyrer, P. (2003), "The value of therapeutic communities in the treatment of personality disorder: a suitable place for treatment?", Journal of psychiatric practice, Vol. 9 No. 4, pp. 291-302.

Shuker, R. (2010), "Forensic Therapeutic Communities: A Critique of Treatment Model and Evidence Base", Howard Journal of Criminal Justice, Vol. 49 No. 5, pp. 463-477.

Shuker, R. and Newton, M. (2008), "Treatment outcome following intervention in a prison-based therapeutic community: a study of the relationship between reduction in criminogenic risk and improved psychological well-being", British Journal Forensic Practice, Vol. 10 No. 3, pp. 33-44.

Taylor, J. (2010), "Psychotherapy for people with learning disabilities: creating possibilities and opportunities. A review of the literature", Journal of Learning Disability and Offending Behaviour, Vol. 1, pp. 15-25. 
Taylor, J., Crowther, S. and Bryant, C. (2015), "Therapeutic communities for people with intellectual disability and complex needs", Adv Mental HIth Intell Disabil, Vol. 9 No. 3, pp. 124-131.

Taylor, J. and Morrissey, C. (2012), "Integrating treatment for offenders with an intellectual disability and personality disorder", The British Journal of Forensic Practice, Vol. 14 No. 4, pp. 302-315.

Torr, J. (2008), "Personality disorder and offending in people with learning disabilities", Adv Mental HIth Learn Disabil, Vol. 2 No. 1, pp. 4-10.

Veale, D., Gilbert, P., Wheatley, J. and Naismith, I. (2014), "A new therapeutic community: Development of a compassion-focussed and contextual behavioural environment", Clinical Psychology and Psychotherapy, Vol. 22 No. 4, pp.285-303.

Warren, F., McGauley, J., Norton, K., Dolan, B., Preedy-Fayers, K., Pickering, A. and Geddes, J. (2003). Review of treatments for severe personality disorder. Available at: http://www.floridatac.com/files/document/rdsolr3003.pdf (accessed 27 September 2015).

Willner, P. (2006), "Readiness for Cognitive Therapy in People with Intellectual Disabilities", Journal of Applied Research in Intellectual Disabilities, Vol. 19 No. 1, pp. 5-16.

Wilson, K., Freestone, M., Taylor, C., Blazey, F. and Hardman, F. (2014), "Effectiveness of modified therapeutic community treatment within a medium-secure service for personality-disordered offenders", The Journal of Forensic Psychiatry \& Psychology, Vol. 25 No. 3, pp. 243261. 
